\title{
A New Process Control Method for Microwave Curing of Carbon Fibre Reinforced Composites in Aerospace Applications
}

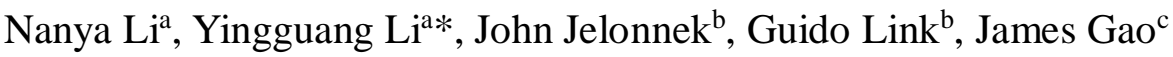

a College of Mechanical and Electrical Engineering, Nanjing University of Aeronautics and Astronautics, Nanjing, 210016, China

b Institute for Pulsed Power and Microwave Technology, Karlsruhe Institute of Technology, Eggenstein-Leopoldshafen,76344, Germany

c Faculty of Engineering and Science, University of Greenwich, Chatham Maritime, Kent, ME4 4TB, UK

\begin{abstract}
For the fabrication of carbon fibre reinforced composites used in aerospace industry, microwave curing technologies are more effective than traditional thermal curing technologies. However, the manufacturer's recommended cure cycles used in autoclave curing are directly adopted into current microwave curing technologies without thorough validation. This paper presents a new methodology of composite microwave curing process control based on cyclic heating and cooling. The heat conduction, residual stress generation and curing kinetic mechanisms during microwave heating composites are revealed. The results of the experiment carried out show significant reduction in residual strain, warpage, total curing time and energy consumption, compared with both traditional thermal curing and current microwave curing technologies. The mechanical properties of samples cured by the new process are compared with the autoclave cured ones.
\end{abstract}

Keywords: A. Polymer-matrix composites (PMCs); B. Residual stress; B. Strength; E. Cure.

* Corresponding author at: College of Mechanical and Electrical Engineering, Nanjing University of Aeronautics and Astronautics, Nanjing, 210016, China. Tel.: +86 25 84895835; fax: +86 2584895906 . 


\section{Introduction}

Carbon fibre reinforced polymer composites with high strength, stiffness and load-bearing/weight ratio have found a wide range of high performance applications [1, 2], such as those in commercial and military aircrafts. Primary load bearing aerospace composites are normally fabricated using traditional thermal curing technologies [3], in which the composite material is placed in an autoclave $[4,5]$, the surrounding air is heated by electric wires which transfer the heat to the material. The main problems of the traditional technology are non-uniform temperature distribution, low curing efficiency, long process cycle, and high energy consumption and cost [6]. For aerospace applications, the most serious limitation is the inability of fabricating composite materials of large and varying thickness, because of the serious temperature gradients induced defects and decrease of performance [7, 8]. More recently, microwave curing technology has been considered as a very attractive alternative to autoclave curing for the fabrication of high performance aerospace composites $[9,10]$.

The advantages of microwave heating compares to conventional heating include: (i) volumetric and selective heating, (ii) fast heating rates, (iii) quick start-up and stopping, (iv) reduction of curing time, (v) saving energy, (vi) higher level of safety and automation, and (vii) friendly to the environment [11]. During microwave curing, energy is supplied by an electromagnetic field directly to the composite material. This results in rapid heating throughout the material thickness with uniform temperature distribution, reduced energy consumption and cost [12], and improved the performance 
of composites [13], compared with traditional thermal curing. Composites cured by microwave had better impact strength than the traditional thermal processed samples [14]. The stiffness of composite samples even post-cured in microwaves were higher than thermal cured ones [15]. The carbon fibre in composite materials can effectively absorb microwaves, the characteristics of microwave heated carbon fibre have been researched and reported significant improve of interface strength of composite [16]. The composite materials with carbon fibre screens were also reported to have strongly absorption properties of microwave in a broadband frequency [17]. Meanwhile, the microwave curing process provided higher joint strength [18], decreased the stresses deformation and total curing time of composite materials [19].

However, in the current microwave curing methods [20], the process parameters of traditional thermal heating (the manufacturer's recommended cure cycles) have been adopted without modifications and thorough validation, such as heating rate, dwell temperature and curing time. The microwave curing mechanisms should be revealed to guide the curing process's design. The experimental results indicated that the microwave heated composite samples consumed shorter cure time by using $2.45 \mathrm{GHz}$ microwave radiation [21]. Microwave was founded to have acceleration effects on the curing rate of polymer resins, but the reaction process of resin is the same with thermal curing [22].

As mentioned above, the microwave curing mechanisms can partly guide the design of curing process parameters. However, due to the complex characteristics, 
multi-physical and inter-coupling influences during microwave curing, there is a need for guidelines to follow in the planning of microwave curing and selection of appropriate technical parameters to take full advantages of microwave curing process, especially in the reduction of residual stresses.

This paper introduces a cyclic heating and cooling process for microwave curing of carbon fibre reinforced composites. For conventional autoclave curing, only the one cooling down during heating process can be achieved, because of the low efficiency heat transfer induced large temperature hysteresis. And the experiment results exhibited that the cure-induced stresses of composites can be slightly reduced [23]. The effects on residual stresses, curing cycle and energy consumption of current microwave curing process, microwave curing with one cooling down and traditional thermal curing process are compared and analyzed. The microwave curing equipment is developed to satisfy the aim of this work. Mechanical strength of different curing processes manufactured composite samples are measured and analyzed.

\section{Theories used in the new microwave curing technology}

\subsection{Microwave heat conduction mechanism}

During traditional thermal curing of carbon fibre composite, heat is conducted via the surrounding air to the surface of the composite in an autoclave, then to the inside of the material. In comparison, during microwave curing, only high absorption materials such as carbon fibre are heated [19]. The heat is conducted from carbon fibre to resin and results in cross-linking reaction of resin, as shown in Fig.1 (a). The composite is 
typically composed of over $60 \%$ volume fraction of carbon fibres. Because the composite is heated directly by microwave, the temperatures of surrounding air and tool material remain relatively low during microwave heating (Fig.1 (b)).

The fast heating rate of composite by microwave leads to strong heat radiation and convection between part, tool and environment. Considering the balance of heat transfer, the microwave heating conduction of composite part can be expressed as [11]:

$\rho_{p} \cdot C_{p} \cdot \frac{\partial T}{\partial t}=k_{x} \frac{\partial^{2} T}{\partial x^{2}}+k_{y} \frac{\partial^{2} T}{\partial y^{2}}+k_{z} \frac{\partial^{2} T}{\partial z^{2}}+\rho_{p} Q_{E}+\rho_{r} Q_{R}$

Where $T$ is curing temperature, $t$ is curing time, $\rho_{p}$ and $\rho_{r}$ are density of composite and resin respectively, $C_{p}$ is the specific heat capacity of composite, $k_{x}$, $k_{y}$ and $k_{z}$ are the composite coefficients of heat conduction at $\mathrm{x}, \mathrm{y}$ and $\mathrm{z}$ directions. $Q_{R}$ is the heat generation rate density of curing reaction, and $Q_{E}$ is the density of the heat generation rate of composite and has the relationship with microwave frequency, the intensity of electric field around composite and the dielectric loss tangent of material [24].

The heat of composite transfer to tool in the $\mathrm{x}, \mathrm{y}$ and $\mathrm{z}$ directions and no heat flow in the symmetrical plane of the composite. In the boundaries of composite and tool, the convective cooling and surface-to-ambient radiation should also be considered. The above equations are the basis for determining the parameters in the proposed new microwave curing process control method.

\subsection{Residual stress induced during microwave curing}

Because of the complex variation of viscoelastic properties of composites, the 
semi-empirical theory was developed to express the stresses induced during microwave curing. Because of the selective heating of microwave, the significant temperature difference between fibre/resin and composite/tool should be considered. Assuming there are interfaces among fibre/resin and composite/tool, respectively, as illustrated in Fig.2 (a). During heating and cooling, the interfaces suffer shear stresses caused by the mismatch of the Coefficients of Thermal Expansion (CTE) of different materials, as shown in Fig.2 (b). The induced stresses caused by the fibre-resin and composite-tool interactions can be expressed as below:

$$
\begin{aligned}
& \sigma_{\text {total }}=\sigma_{\text {fiber-reisn }}+\sigma_{\text {composite-tool }} \\
& \sigma_{\text {fiber-reisn }}=\Delta T_{f r} \beta_{f} E_{f}-\sigma_{r}(\tau) \\
& \sigma_{\text {composite-tool }}=\Delta T_{c t}\left(\beta_{c} E_{c}(t)-\beta_{t} E_{t}\right)
\end{aligned}
$$

Where $\beta_{f}, \beta_{t}$ and $\beta_{c}$ are the CTEs of carbon fibre, tool and composite respectively. $E_{f}, E_{t}$ and $E_{c}(t)$ are the Young modulus of carbon fibre, tool and composite, respectively. $\sigma_{r}(\tau)$ represents the stress in the resin related to the viscoelastic dynamics. $\Delta T_{f r}$ is the temperature difference between fibre and resin. $\Delta T_{c t}$ is the temperature difference between composite and tool. Eq.3 represent that the shear stresses in fibre/resin interface induced by the stresses of fibre thermal expansion and resin viscoelastic with different direction. $E_{c}(t)$ is the Young modulus of composite related with curing time, the calculation equation is shown in Eq.6. The linear viscoelastic properties of the resin aroused by temperature change can be represented by the reduced time and shift factor as Eq.5 [25]. As shown in Fig. 3 (a). 
The spring and dashpot portions on behalf of the elastic and viscosity properties of resin during cure, respectively. The transient modulus of composite can be expressed as below:

$\sigma_{r}(\tau) \propto \Gamma\left[\kappa_{f}(\tau), \xi(t)-\xi(\tau)\right]$

$E_{c}(t)=E^{\prime}(t)+i E^{\prime \prime}(t)$

Where, $\Gamma\left[\kappa_{f}(\tau), \xi(t)-\xi(\tau)\right]$ is the time dependent viscoelastic module, which includes shift factor $\left(\kappa_{f}(\tau)\right)$ and reduced time $(\xi(t))$. The shift factor and reduced time are both related to the loss tangent of polymer resin. The loss tangent value can be calculated by the ratio of loss modulus $\left(E^{\prime \prime}(t)\right)$ and storage modulus $\left(E^{\prime}(t)\right)$, which reflect the viscosity and elastic properties respectively. The temperature variation frequency and amplitude are both related to the thermal mechanical loss tangent of resin.

During the curing process, the phase transition of matrix resin is represented by the curve of dynamic thermal mechanical loss tangent-temperature, as show in Fig.3 (b). At the beginning of curing, the resin is sol liquid with an infinity value of loss modulus. As the temperature rises, it starts to gel, and the phase changes from sol-gelled rubber state to totally cured gelled glass state. Before the gelation of resin, the accumulated stress in fibre-resin and composite-tool can be relaxed, because of the high viscosity property. However, after gelation, the resin starts to store stress due to the viscoelastic properties of sol-gelled rubber phase [25]. Because of the constraint of the composite by the outer vacuum pressure and tool friction, the elastic deformation cannot release instantaneously, thus there is delayed elastic response to elastic deformation. The 
hysteresis of elastic deformation leads to the stress accumulation of composite during the continuous curing process. Because of the chemical reaction of resin, the stress will be 'locked' in the composite until curing completed and resulting in warpage of the composite.

To release the 'locked' stress, a new cyclic heating and cooling method based on the microwave heat conduction mechanism is proposed and shown in Fig.4. $g(t)$ is the temperature variation of composite under the cyclic heating-cooling process. Compare with the traditional autoclave curing methods, the curing temperature in the reaction process remain invariable (dwell stage), or only one cooling down and re-heating can be achieve [23]. The reason can be explained as the temperature of surrounding air (large heat capacity) and tooling are higher than the composite in the oven and give rise to the bad control ability of composite temperature. According to Eq.1, the strong heat dissipation of composite can lead to fast cooling down rate. Meanwhile, the volumetric heating of microwave provided fast heating rate of composite. The symbols in Fig.4, $P(t), A(t)$ and $\varphi(t)$ denote the temperatures at the equilibrium point, amplitude and the frequency respectively. Through cyclic heating and cooling, the stress ( $\tau_{h-1}$ and $\tau_{h-2}$ ) 'locked' in the previous stage (e.g., heating in Fig.2 (b)) will be counter-acted in the next stage (e.g., cooling in Fig.2 (b)) by the same value but opposite direction stresses ( $\tau_{c-1}$ and $\left.\tau_{c-2}\right)$.

\subsection{Microwave curing kinetics}

For the purpose of controlling the curing process and ensuring the complete curing 
of composites, the microwave curing kinetics of composite should be studied. As microwave heating does not change the structures of the cured products [26], the methods for analyzing the kinetics of the traditional thermal heating are still applicable. The degree of curing composite is denoted as $\alpha$, which is the ratio of the extent of exothermic reaction at certain curing temperature, and the fully completed exothermic reaction. According to the Arrhenius law [27], the microwave curing kinetics of reaction is expressed as:

$d \alpha / d t=K \exp \left(-E_{a} / R T\right)\left(B+\alpha^{m}\right)(1-\alpha)^{n}$

Where, $K$ is the frequency factor, $E_{a}$ is the activation energy, $R$ is the universal gas constant, $B$ is a temperature independent parameter, $m$ and $n$ are the orders of the autocatalytic and non-catalyzed polymerization reactions, respectively. For microwave curing kinetics, the activation energy $E_{a}$ is lower than for the conventional thermal curing, thus higher curing rates in a microwave process can be expected [28]. At the beginning of curing, the reaction rate is very low. After the curing temperature reaches a certain level, the reaction rate accelerates fast to arrive the gelation point. Gelation is a sudden and irreversible transformation of resin from a viscous liquid to an elastic gel glass. The cyclic heating-cooling process leads to the variation of curing rate. The curing rate at the heating stage is the fastest, and is the lowest in the cooling stage.

\section{New process control method for microwave curing}

Instead of directly using the manufacturer's recommended cure cycles of traditional thermal process as curing parameters in the current microwave curing 
technologies, the proposed new method is based on the cyclic heating-cooling process as illustrated in Fig.4, which reduces the cure-induced stress through the alternating stress counter-acting of repeating heating and cooling during the curing process. The duration period of heating and cooling determine the temperature variation parameters of cyclic curve (amplitude $A(t)$ and frequency $\varphi(t)$ ) shown in Fig. 4. For example, the stresses 'locked' in the heating stage will be calculated based on the semi-empirical equations of Eq. 3 and Eq. 4. Then, the calculated stresses can be devoted to inversely solve the above equations and acquire almost the same value but opposite direction stresses that will be applied in the cooling stage. It is noted that at the inverse solution process, the degree of curing and viscoelastic properties of composite were already changed. Thus, the cooling down process will not be the same with the heating stage. In current microwave curing methods, there is slightly temperature fluctuation in the dwell stage. Because of the heat strongly dissipates from composite to surrounding environment and leads to difficulties of temperature control. However, the new microwave curing method actively controls the fluctuation of temperature during the 'dwell' stage in order to reduce cure-induced stresses. Traditional autoclave curing with quick heating rate normally results in uneven temperature distribution in the composite [29]. In comparison, the fast heating rate of microwave curing can maintain a more even temperature distribution across the composite and the tool, and can reduce residual stress [19]. The results of the experiment carried out in this project indicated that fast cooling rates using the new microwave curing method can further reduce the induced 
stress. Therefore, heating rate $H_{r}$ and cooling rate $C_{r}$ should be controlled within the maximum heating and cooling capability of the microwave curing equipment. The long dwell period in current microwave curing methods can be reduced, as there is no need for stress relaxation by long dwell time in the new cyclic heating-cooling method. The dwell temperature and time parameters change with time, and can be expressed as:

$$
\begin{aligned}
& H_{r} \times t_{\text {heating }}+D_{t} \geq t_{\alpha=1.0} \\
& P(t)+A(t) / 2 \leq T_{\text {char }}
\end{aligned}
$$

Eq. 8 means that the degree of curing should arrive $100 \%$ before the final cooling process at least, without the need for a long dwell period for relaxing stress. Eq.9 means that the maximum heating temperature cannot exceed the char temperature of resin $\left(T_{\text {char }}\right)$. In order to improve the quality of composite, the excessive resin in the prepreg will be extruded at the lowest viscosity where the holding time is $R_{t}$. Fig.5 shows the whole microwave curing cycle with technical parameters using the new cyclic heating-cooling method for composites that meet the high requirements of aerospace applications.

After the first (Heating) stage and when the resin reaction rate reaches the highest values, temperature $T_{p}$ at that point should be 'maintained' (but with controlled cyclic variation) to allow resin extrusion for improving the compaction degree until the gelation point. The controlled temperature variation before the gelation point aims to release any stress accumulated during the first Heating stage, and to control the cross-linking reaction rate. To ensure no new stress is induced, the temperature should 
be reduced, from the gelation point to the temperature $\left(T_{d \alpha / d t=0}\right)$ at which the resin reaction rate is zero. The next heating stage is to rekindle the curing reaction, and the following cooling stage will counteract the stress accumulated during the preceding heating stage. The cyclic heating-cooling process continues until the composite reaches $100 \%$ curing degree, at temperature $T_{d}$. As shown in Fig.5, the amplitude values exponentially decay with the increase of curing time, because of the exponential increasing variation of the mechanical loss tangent and curing degree of composite.

Since the thickness of the composite is by far smaller than the other two dimensions, the stress imposed by the tool along the thickness direction of composite is ignored. The temperature amplitudes of every heating-cooling cycle were determined by the residual stress accumulated in the preceding cycle. For the frequency of the heating-cooling cycle, its relationship with resin property and shift factor can be expressed as [30]:

$$
\log \varphi(t)=\log f_{r e f}(t)+\log \kappa_{f}(t)
$$

Where, $f_{\text {ref }}(t)$ is the characteristic frequency of resin, and can be represented as the thermal mechanical loss tangent. According to Eq.5 and Eq.6, the frequency and amplitudes change with the viscoelastic properties of resin exponentially and can effectively release the stress locked in the composite.

\section{Materials and experiment methods}

Carbon fibre reinforced bismaleimide composite samples of 200mm x 200mm x 2.3mm size were fabricated by ply 18 plies of T700/QY9611 (carbon fibre T700 
reinforced bismaleimide resin QY9611) unidirectional prepreg with the matrix volume fraction of $35.7 \%$. This kind of prepreg has high usage temperature and strength, usually applied in primary load bearing composites in high-speed fighter planes. The ply sequence is $\left[02 /-45 / 0 / 45 / 0 / 90 / 0_{4} / 90 / 0 / 45 / 0 /-45 / 0_{2}\right]$ and generally used in parts bearing tensile loads. For comparison, the composite samples were fabricated using the traditional thermal curing, the current microwave curing, the microwave curing with one cooling down and the proposed new microwave curing technology respectively. Each curing technologies tested three different samples respectively and the statistical results were analyzed.

Experiment using the new method is shown in Fig.6 (a). Three Fibre Bragg Grating (FBG) sensors are embedded in the middle of the sample to measure the strains during curing (note: strains can be converted to residual stress) [31, 32]. The aluminium foil is stuck on the edge of the prepreg to avoid arcing of carbon fibre, and a capillary tube is applied to compensate the temperature change according to the reading of the FBG sensor as shown in Fig.6 (b). The air tube is connected to the compressed air bag to control the cooling rate. The $2.45 \mathrm{GHz}, 20 \mathrm{KW}$ octagon microwave chamber (designed by the authors), developed with Labview-PCI data acquisition and control system, was used to cure the composite sample with uniform temperature distribution. Two no-metal temperature sensors are placed on the surface of a vacuum bag to measure the microwave curing temperature. The mechanical strength of composite samples were measured by using MTS C45 electronic universal testing machine and the ZEISS 
EVO18 scanning electron microscope was applied to observe the fracture surface of tested samples (surface gold plating).

For the sample using the new microwave curing technology, the microwave curing process was designed based on the curing kinetic, differential scanning results and the thermal mechanical loss tangent of unidirectional T700/ QY9611 laminate (tested by the authors [33]). According to the Flory's theory of gelation, the BMI resin usually has a gelation point at about 0.6 of curing degree.

\section{Discussion of Results}

The samples for traditional thermal curing were heated in an autoclave with the same vacuum pressure and conditions of microwave curing. The autoclave heating process followed the manufacturer's recommended cure (MRC) cycle. The samples for current microwave curing were heated using parameters basically the same as traditional thermal curing, except that the dwell time at $200^{\circ} \mathrm{C}$ was cut down, because of the fast microwave curing rate. The comparison of measured curing temperature curve of a set of the experimental samples can be seen in Fig.7. The new microwave curing process parameters are shown in Fig.8, which zoomed portion of the red curve in Fig.7. The microwave curing process with one cooling down has only one cooling-heating cycle at the gelation point (cooling down to $130^{\circ} \mathrm{C}$ after dwell at $185^{\circ} \mathrm{C}$ ) and tested to verify the effectiveness of the new microwave curing. It is clear that the new microwave curing method reduced the total curing time to $45 \%$ of the traditional autoclave curing. In order to verify the reliability of the results, three samples of each curing technologies 
are tested respectively and the statistical results are shown in Fig. 9 and Fig. 10. The measured strains with standard deviations corresponding to the temperature of different curing process are shown in Fig.9. In Fig. 10, the residual stress converted from the residual strain and modulus of totally cured composite (200GPa) are compared with standard deviations. The experiment results indicated that the strains change with the curing temperature. After the curing process, the degree of curing of the composite samples was tested, and $100 \%$ curing degree have been achieved for all samples.

As shown in Fig. 9, the demould strains of the displayed set of samples were measured when they were demoulded from the tool and marked with solid dots in the figure. Then, the statistical residual strains were obtained after 12 hours of demoulding (placed at room temperature with no constraint) and marked in 1320 minute in Fig. 9. The measured stress of the sample cured by the new microwave curing technology was positive (tensile), whilst the stresses of the samples cured by the traditional thermal, current microwave curing and microwave curing with one cooling down are negative (compressive), as shown in Fig. 10. The compressive stresses of the samples cured by traditional thermal and current microwave methods increased after 12 hours - this is caused by the release of tool imposed stresses. However, for the sample cured by the new method, the tensile stress is further reduced since the compressive stresses of tool were released after 12 hours. The measurement results shown in Fig. 10 of four different curing processes exhibit that the maximum warpage of the new microwave curing technology is smaller than other curing process. The interesting results exhibit that the 
microwave curing with one cooling down has lower strain but higher warpage than current microwave curing. The reason may be due to the tool-part interaction.

As shown in Table 1, the mechanical strength of three different curing processes are measured according to American Society for Testing Material (ASTM) standards. The tensile strength of the new microwave cured samples are lower than the current microwave and autoclave cured ones. The flexural strength and tensile modulus of microwave cured samples are higher than the autoclave cured ones. In order to analyzing the reasons, the SEM micrographs of samples cured by different process showing the fracture surface after tensile and three-point flexural test are illustrated in Fig.11. For the tensile strength, the fracture surface of tensile test samples have different amount of residue resin. The new and current microwave process cured sample has more residue resin on the surface of fibre. The autoclave cured sample has a relatively clean fibre fracture section. This indicated that the microwave cured composites have stronger interface bonding than the autoclave cured one. However, the strong interfacial strength may not benefit the tensile strength of composite. The tensile test mainly reflects the fibre strength and the tensile strength of carbon fibre may decreased by long time microwave irradiation [16]. Because of the graphite on the surface is etched to form grooves by microwave radiation and the grooves can reduce the fibres' strength.

The tensile modulus mainly depends on the toughness of resin matrix, and the microwave cured resin has higher tensile modulus than autoclave cured one [35]. As the cyclic microwave heating and cooling process, the curing reaction also occurs cyclic 
acceleration and deceleration phenomenon. This can affect the curing of resin and reduce the influence of microwave on resin reaction in the cooling down stage, lead to lower tensile modulus of composite. Fig. 11(b), (d) and (f) exhibit the breakage section after flexural test of new microwave process, current microwave process and autoclave cured samples, respectively. The microwave cured samples have more resin on the carbon fibres compare with the autoclave cured samples. More clean fibres and smooth grooves of resin can be founded on the breakage section of autoclave cured samples. It is known that the flexural strength of composites are determined by the interfacial strength between fibre and resin [36]. The previous research exhibited that microwave curing process can effectively improve the interfacial strength between carbon fibre and matrix resin $[20,37]$. The reason is that the chemical mechanism of the fiber/matrix interface is essentially identical for microwave and thermal curing, but the selective heating of microwave leads to the prioritized heating of carbon fibre and can significantly increase the interfacial strength. However, for the new microwave process, the amount of heat conduct from fibre to resin during the cooling stage and decreased the selective heating benefits of microwave. Therefore, the new cyclic heating and cooling microwave curing process can effectively release the cure-induced stresses of composite, but may reduce the interfacial strength of fibre and resin compared with the traditional microwave curing process.

The tensile strength above $1200 \mathrm{Mpa}$ can satisfy the requirements of airplane. However, the engineers desire to obtain high flexural strength and appropriate tensile 
modulus (from $150 \mathrm{GPa}$ to $170 \mathrm{GPa}$ ) to cover the shortage of composite parts. Thus, the new microwave curing process has enormous potential in aerospace applications.

Through recording the electrical power of the three experiments, the energy consumption results are: for the same volume of curing chamber (2000L), the energy consumption is $52 \mathrm{Kwh}$ for the new microwave curing, $105 \mathrm{Kwh}$ for current microwave curing, and $1800 \mathrm{Kwh}$ for autoclave (1m diameter and $2 \mathrm{~m}$ length) curing respectively. This means that the energy consumption of the new microwave curing technology is only $3 \%$ of the traditional autoclave curing, and $50 \%$ of current microwave curing.

\section{Conclusion}

This paper presented a new microwave curing technology based on a cyclic heating-cooling process control method by considering the special characteristics of microwave curing. The experimental results demonstrated that: (i) cure-induced residual strains and warpage of composites can be significantly reduced by the new microwave curing process; (ii) total curing time can be reduced to $45 \%$ of traditional thermal curing and $56 \%$ of current microwave curing technologies and (iii) energy consumption can be reduced to $3 \%$ of autoclave curing and $50 \%$ of current microwave curing. The flexural strength and tensile modulus of samples cured by the new microwave curing process are higher than the autoclave cured samples, and the mechanisms were analyzed.

Preliminary industrial validation has been carried out for primary load bearing aerospace composites. Further work is to develop recommendations and guidelines for 
using the new method and select technical parameters.

\section{Acknowledgements}

This work was supported by the National Natural Science Foundation of China [grant numbers 51575275]. International collaboration has also been supported by the above grants. The authors sincerely appreciate the continuous support provided by industrial collaborators.

\section{Reference}

[1] Gao L, Thostenson E T, Zhang Z, Chou T W. Coupled carbon nanotube network and acoustic emission monitoring for sensing of damage development in composites. Carbon 2009;47(5): 1381-1388.

[2] Liu D, Zhu Y, Ding J, Lin X, Fan X. Experimental investigation of carbon fiber reinforced poly (phenylenesulfide) composites prepared using a double-belt press. Composites Part B 2015;77: 363-370.

[3] Edwards K L, A risk-based approach to manufacturing process control: use in autoclave moulded composite sandwich panels, Materials and Design 2005;26: 690-699.

[4] Yoo S, Han M, Hong J, Chang S. Simulation of curing process of carbon/epoxy composite during autoclave degassing molding by considering phase changes of epoxy resin. Composites Part B 2015;77: 257-267.

[5] Yanagimoto J, Ikeuchi K. Sheet forming process of carbon fiber reinforced plastics for lightweight parts, CIRP Annals-Manufacturing Technology 2012;61(1): 247-250. 
[6] Takagaki K, Hisada S, Minakuchi S, Takeda N. Process improvement for out-of-autoclave prepreg curing supported by in-situ strain monitoring. Journal of Composite Materials 2016; DOI: 10.1177/0021998316672001

[7] Sorrentino L, Esposito L, Bellini C. A new methodology to evaluate the influence of curing overheating on the mechanical properties of thick FRP laminates. Composites Part B: Engineering 2016. DOI:10.1016/j.compositesb.2016.10.064.

[8] Li Y, Li N, Gao J. Tooling design and microwave curing technologies for the manufacturing of fiber-reinforced polymer composites in aerospace applications. The International Journal of Advanced Manufacturing Technology 2014;70(1-4): 591-606.

[9] Kwak M, Robinson P, Bismarck A, Wise R. Microwave curing of carbon-epoxy composites: Penetration depth and material characterisation. Composites Part A: Applied Science and Manufacturing 2015;75: 18-27.

[10]Papargyris D A, Day R J, Nesbitt A, Bakavos D. Comparison of the mechanical and physical properties of a carbon fibre epoxy composite manufactured by resin transfer moulding using conventional and microwave heating. Composites Science and Technology 2008;68(7): 1854-1861.

[11]Feher L E. Energy Efficient Microwave Systems: Materials Processing Technologies for Avionic, Mobility and Environmental Applications. Springer Science \& Business Media 2009. pp. 54-55.

[12] Thostenson E T, Chou T W. Microwave processing: fundamentals and applications. Composites Part A: Applied Science and Manufacturing 1999;30(9): 1055-1071.

[13] Chaowasakoo T, Sombatsompop N. Mechanical and morphological properties of fly ash/epoxy composites using conventional thermal and microwave curing methods. Composites Science and Technology 2007;67(11): 2282-2291.

[14] de Vergara U L, Sarrionandia M, Gondra K, Aurrekoetxea J. Impact behaviour of basalt fibre reinforced furan composites cured under microwave and thermal conditions. Composites Part B: Engineering 2014;66: 156-161. 
[15]H. Ku, M. Prajapati, F. Cardona Thermal properties of sawdust reinforced vinyl ester composites post-cured in microwaves: A pilot study Composites: Part B, 2011;42: 898-906.

[16] Wang B, Duan Y, Zhang J, Zhao X. Microwave radiation effects on carbon fibres interfacial performance. Composites Part B: Engineering 2016;99: 398-406.

[17]Zang Y, b, Xia S, Lia L, Ren G, Chen Q, Quan H, Wu Q, Microwave absorption enhancement of rectangular activated carbon fibers screen composites Composites Part B: Engineering 2015; 77: 371-378.

[18]Bajpai P K, Singh I, Madaan J. Joining of Natural Fiber Reinforced Composites Using Microwave Energy: Experimental and Finite Element Study. Materials and Design 2012;35: 596-602.

[19] Li N, Li Y, Hao X, Gao J. A Comparative Experiment for the Analysis of Microwave and Thermal Process Induced Strains of Carbon Fiber/bismaleimide Composite Materials. Composites Science and Technology 2015;106: 15-19.

[20] Kwak M. Microwave curing of carbon-epoxy composites: process development and material evaluation, Imperial College, London, 2016.

[21]Chaowasakoo, T., \& Sombatsompop, N. Mechanical and morphological properties of fly ash/epoxy composites using conventional thermal and microwave curing methods. Composites Science and Technology 2007;67(11): 2282-2291.

[22] de Vergara U L, Sarrionandia M, Gondra K, Aurrekoetxea J. Polymerization and curing kinetics of furan resins under conventional and microwave heating. Thermochimica Acta 2014;581: 92-99.

[23] Kim S S, Murayama H, Kageyama K, Uzawa K, Kanai M. Study on the curing process for carbon/epoxy composites to reduce thermal residual stress. Composites Part A: Applied Science and Manufacturing 2012;43(8): 1197-1202.

[24]Farag S, Sobhy A, Akyel C, Doucet J, Chaouki J. Temperature profile prediction within selected materials heated by microwaves at $2.45 \mathrm{GHz}$. Applied Thermal Engineering 2012;36: 360-369. 
[25] O'Brien D J, Mather P T, White S R. Viscoelastic properties of an epoxy resin during cure. Journal of Composite Materials 2001;35(10): 883-904.

[26] Johnston K, Pavuluri S K, Leonard M T, Desmulliez M P Y, Arrighi V. Microwave and thermal curing of an epoxy resin for microelectronic applications.

Thermochimica Acta 2015;616: 100-109.

[27] de Vergara U L, Sarrionandia M, Gondra K, Aurrekoetxea J. Polymerization and curing kinetics of furan resins under conventional and microwave heating. Thermochimica Acta 2014;581: 92-99.

[28] Tanikella R V, Allen S A B, Kohl P A. Variable-frequency microwave curing of benzocyclobutene. Journal of Applied Polymer Science 2002;83(14): 3055-3067.

[29] Nielsen M W, Schmidt J W, Høgh J H, Waldbjørn J P, Hattel J H, Andersen T L, Markussen C M. Life cycle strain monitoring in glass fibre reinforced polymer laminates using embedded fibre Bragg grating sensors from manufacturing to failure. Journal of Composite Materials 2014;48(3): 365-381.

[30] Knapp G, Oreski G, Pinter G. Method to characterize the damping behavior of thin passively constrained layer laminates using dynamic mechanical analysis (DMA) in shear mode. Polymer Testing 2015;42: 215-224.

[31] Kim H S, Yoo S H, Chang S H. In situ monitoring of the strain evolution and curing reaction of composite laminates to reduce the thermal residual stress using FBG sensor and dielectrometry. Composites Part B: Engineering 2013;44(1):446-452.

[32] Antonucci V, Esposito M, Ricciardi M R, Giordano M, Zarrelli M. Strain monitoring of composite elements by fibre Bragg grating sensors during a quasi-static indentation. Composites: Part B 2014;56: 34-41.

[33]Li N, Li Y, Zhang L, Hao X. Kinetics modeling of carbon-fiber-reinforced bismaleimide composites under microwave and thermal curing. Journal of Applied Polymer Science 2016;133(33):1-8. DOI: 10.1002/APP.43770.

[34]Zhang K, Gu Y, Li M, Zhang Z. Effect of rapid curing process on the properties of carbon fiber/epoxy composite fabricated using vacuum assisted resin infusion 
molding. Materials and Design 2014;54: 624-631.

[35] Chew C S, Ku H, Baddeley D, Snook C. Micrographs of the fracture of vinyl ester composites cured by microwaves: pilot study. Journal of electromagnetic waves and applications 2005;19(1): 67-82.

[36] Wang X, Zhang J, Wang Z. Effects of interphase properties in unidirectional fiber reinforced composite materials. Materials and Design 2011;32(34):86-92.

[37]Zhou J, Li Y, Li N, Hao X, Liu C. Interfacial shear strength of microwave processed carbon fiber/epoxy composites characterized by an improved fiber-bundle pull-out test. Composites Science and Technology, 2016;133:173-183. 


\section{Artwork and Tables with Captions}

Fig.1. (a) Heat conduction between carbon fibre and resin; (b) Heat conduction between the composite and surrounding air and tool.

Fig.2. (a) Assumed Interfaces between fibre-resin and composite-tool; (b) Changes in shear stress direction during heating and cooling.

Fig.3. Viscoelasticity of resin during curing. (a) the viscosity properties of resin during cure; (b) mechanical loss tangent variation of resin during heating.

Fig.4. The proposed new cyclic heating and cooling method.

Fig.5. The parameters of the new microwave curing method for composites for aerospace applications.

Fig.6. (a) Sample preparation with FBG sensors; (b) Microwave curing in an octagon microwave chamber.

Fig.7. The comparison of measured temperature variations of four curing technologies.

Fig.8. Zoomed portion of the curve in Fig.7 for the new microwave curing process showing parameters.

Fig.9. The measured strains of samples cured by four process technologies.

Fig.10. Comparison of residual stresses and maximum warpage of different curing technologies.

Fig.11. SEM micrographs of samples cured by different process showing the fracture surface after tensile and three-point flexural test. (a) and (b) tensile and flexural test samples cured by new microwave process; (c) and (d) tensile and flexural test samples cured by current microwave process; (e) and (f) tensile and flexural test samples cured by autoclave.

Table 1. Mechanical strength of three different curing processes. 